\title{
Added Value of Diffusion-Weighted Magnetic Resonance Imaging in Differentiating Musculoskeletal Tumors Using Sensitivity and Specificity: A Retrospective Study and Review of Literature
}

\author{
Deb K. Boruah ${ }^{1,2}$, Bidyut Gogoi ${ }^{3}$, Ruchi S. Patni ${ }^{2}$, Kalyan Sarma ${ }^{4}$, Karuna Hazarika ${ }^{1}$ \\ 1. Radiodiagnosis, Tezpur Medical College, Tezpur, IND 2. Radiodiagnosis, Assam Medical College, Dibrugarh, IND 3. \\ Pathology, Assam Medical College, Dibrugarh, IND 4. Radiology, North Eastern Indira Gandhi Regional Institute of \\ Health and Medical Sciences, Shillong, IND
}

Corresponding author: Deb K. Boruah, debpal80@gmail.com

\begin{abstract}
Background: Diffusion-weighted imaging (DWI) provides added value to conventional MRI imaging in diagnosing and differentiating various benign and malignant musculoskeletal tumors.
\end{abstract}

Objective: The study aims to evaluate the diagnostic efficacies of diffusion-weighted imaging along with the conventional MRI sequences for differentiating benign and malignant musculoskeletal tumors using sensitivity and specificity.

Materials and methods: This retrospective study was carried out on 73 histopathologically proven patients of various musculoskeletal tumors who presented to a tertiary care center between March 2017 to October 2018. Relevant clinical examinations and MRI scan of the requested body part of the musculoskeletal system were performed. Mean apparent diffusion coefficient (ADC) values were calculated in the bone as well as soft tissue tumors after placing uniform-sized region of interest (ROI) in the non-necrotic portion of the tumor.

Review began 11/28/2020 Review ended 12/31/2020 Published 01/01/2021

๑) Copyright 2021

Boruah et al. This is an open access article distributed under the terms of the Creative Commons Attribution License CC-BY 4.0., which permits unrestricted use, distribution, and reproduction in any medium, provided the original author and source are credited.
Statistical analysis: Independent t-test and one-way analysis of variance (ANOVA) test were used to compare the mean $\mathrm{ADC}$ values of the various tumors with the histopathology. Receiver operating characteristic (ROC) curve analysis was done to determine the cut-off mean ADC values in the various bone and soft tissue tumors.

Results: Of 73 patients with musculoskeletal tumors (benign=20, malignant $=53$ ), 47 patients were bone tumors (benign=12, malignant=35) and 26 patients were soft tissue tumors (benign=eight, malignant $=18$ ). Mean ADC value of benign bone tumor was $1.257 \pm 0.327[\mathrm{SD}] \times 10^{-3} \mathrm{~mm} 2 / \mathrm{s}$ and malignant was $0.951 \pm$ $0.177[\mathrm{SD}] \times 10^{-3} \mathrm{~mm} 2 / \mathrm{s}$. The mean ADC value of benign soft tissue tumor was $1.603 \pm 0.444[\mathrm{SD}] \times 10^{-3} \mathrm{~mm} 2 / \mathrm{s}$ and malignant was $1.036 \pm 0.186[\mathrm{SD}] \times 10^{-3} \mathrm{~mm} 2 / \mathrm{s}$. The cut-off mean ADC value was $1.058 \times 10^{-3} \mathrm{~mm} 2 / \mathrm{s}$ for differentiating benign from malignant bone tumor with a sensitivity of $83.3 \%$, specificity of $66.7 \%$ and accuracy of $78.7 \%$ while the cut-off mean ADC value of $1.198 \times 10^{-3} \mathrm{~mm} 2 / \mathrm{s}$ for differentiating benign from malignant soft tissue tumors with a sensitivity of $83.3 \%$, specificity of $87.5 \%$ and accuracy of $84.6 \%$.

Conclusions: DWI with ADC mapping can be used as an additional reliable tool along with conventional MRI sequences in discriminating benign and malignant musculoskeletal tumors.

Categories: Radiation Oncology, Radiology, Orthopedics

Keywords: diffusion-weighted imaging (dwi), apparent diffusion coefficient (adc), bone tumor, soft tissue tumor

\section{Introduction}

MRI is one of the imaging modalities of choice to detect intramedullary bony abnormality even with a negative bone scan [1]. Because of excellent soft-tissue contrast and multiplanar imaging capability, the conventional MRI sequences can delineate tumor size, margins, locations, tumor necrosis, and neurovascular bundle involvement, tumoral heterogeneity and adjacent joint involvement [2]. However, adding other MRI sequences like diffusion-weighted imaging (DWI), dynamic contrast-enhanced MRI (DCEMRI), and MR spectroscopy helps to more accurately differentiate benign from malignant bone as well as soft tissue tumors [3-4].

DWI is a functional MRI technique based on the Brownian movement of water molecules where the apparent diffusion coefficient (ADC) quantifies the Brownian movement. The presence of cellular membranes limits the diffusion in a highly cellular microenvironment, resulting in low ADC value while free diffusion takes 
place in an acellular region resulting in high ADC value [5]. Because of this property, DWI is able to provide both qualitative and quantitative assessments of intra-tumoral cellularity [6]. Various literature have shown the added advantage of DWI and ADC mapping over the conventional MRI sequences in differentiating various musculoskeletal tumors, diffuse bone marrow infiltrative lesions, benign and pathological vertebral collapse [6-8]. DWI with ADC mapping is also utilized for assessment of the therapeutic response after treatment of various musculoskeletal or diffuse marrow infiltrative lesions [6-8]. Tumor response to radiotherapy or chemotherapy showed higher ADC values as compared with the pre-treatment ADC value [9]. DWI can be a reliable additional tool over conventional MR findings in differentiating different types of bone as well as soft tissue tumors [3,10-11]. In certain situations, conventional MRI fails to differentiate benign from malignant musculoskeletal tumors, where quantitative DWI, DCE-MRI, and MR spectroscopy play an important role [2-3,12-13]. Because of the overlapping of ADC values in the benign and malignant musculoskeletal tumors, tumor differentiation is complicated in a few situations as ADC values are affected by tumor cellularities and extracellular substances [14]. Previous studies concluded that DWI can be used as an adjunct imaging to conventional MRI sequences for characterization and differentiation of various musculoskeletal tumors [14-15].

The study aims to evaluate the diagnostic efficacies of diffusion-weighted imaging along with the conventional MRI sequences for differentiating benign and malignant musculoskeletal tumors using sensitivity and specificity.

\section{Materials And Methods}

\section{Study design}

A hospital-based retrospective study was conducted in a tertiary care hospital on 73 histopathologically confirmed patients with musculoskeletal tumors over for 18 months from March 2017 to October 2018. This retrospective study was approved by Institutional Ethics Review Committee.

Seventy-three histopathologically confirmed patients with various musculoskeletal tumors were included in this study after using the following exclusion criteria: 1. Benign soft tissue tumors like lipoma,

angioleiomyoma, tenosynovial giant cell tumors were excluded because of false positivity due to their low $A D C$ value. 2. Vertebral lesions. 3. Lesions with a diameter of less than $1 \mathrm{~cm}$ were excluded because of difficulties in placing a region of interest (ROI) for the measurement of ADC values. 4. Benign cartilaginous tumors like osteochondroma and enchondroma due to their high ADC value.

\section{Test methods}

All patients underwent an MRI examination using a 1.5 T MR scanner (Magnatom Avanto; Siemens Medical Systems, Erlangen, Germany). An appropriate body or surface coil was used, depending upon the location and size of the lesion.

Various MRI sequences are shown in Table 1. 


\section{Cureus}

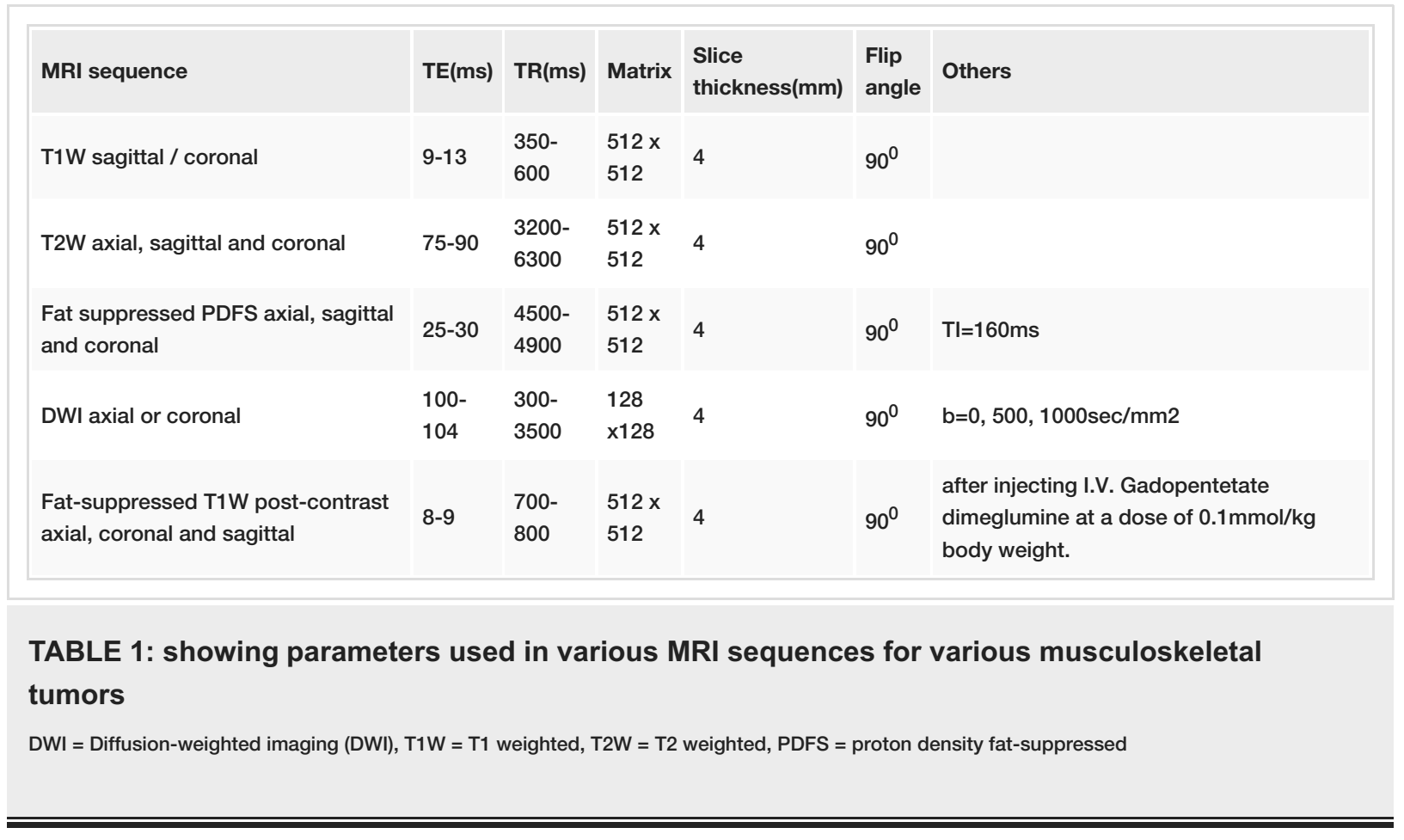

\section{Analysis}

Two radiologists retrospectively reviewed the MR images. During MR image analysis, the two radiologists were blinded to the clinical history or previous radiological reports of the studied patients. The size and ADC values were measured independently by the two radiologists and the mean values were used for the results.

\section{Analysis of Conventional MR Images}

We evaluated the following characteristics of a lesion like tumor sizes, margins, locations, neurovascular bundle involvement, peri-tumoral edema, tumor heterogeneity, and tumor necrosis. Tumor size was obtained from the largest dimension of a tumor. Margins of tumor classified into well defined, partially illdefined, and ill-defined. A "well defined" margin was considered when the margin of a tumor was differentiated from surrounding structures regardless of peritumoral edema. A "partially ill-defined” was classified when the margin of a tumor was mostly well defined. The location of a tumor was classified as superficial when involving skin and subcutaneous tissue or deep when the tumor was located deeper in the deep fascia. The presence of bone involvement was confirmed when there was bony cortical erosion or medullary canal involvement. Peri-tumoral edema was defined where there were peri-tumoral bright T2 weighted images (T2WI) or proton density fat-suppressed (PDFS) hyperintensities. Tumor heterogeneity was defined with mixed-signal intensities on T1WI or T2W images. Tumor necrosis was defined as an area of T2WI or short tau inversion recovery (STIR) hyperintensities that were not enhanced on post-contrast images.

\section{ADC Calculation Analysis}

ADC values were generated on a pixel by pixel basis. Minimum, maximum and mean ADC values were calculated from placing either round or elliptical ROIs, however, mean ADC values were selected for statistical analyses. ADC values were expressed in 10-3 x mm2/second. We measured the ADC value in the operating system console using multiple uniform sizes (area, minimum $10 \mathrm{~mm} 2$, maximum $50 \mathrm{~mm} 2$ ) at least six ROIs, where three ROIs were placed in the central non-necrotic portion of the tumor and another three in the peripheral portion. Usually, the ROI was selectively placed in the solid, enhancing, non-necrotic, and/or DWI restricted regions of a tumor. The ROI position was always checked with reference to the conventional MRI images to avoid contamination from adjacent normal-appearing bone or soft tissue. The areas of artifacts, image distortions, partial volume effect, and most peripheral margin of a tumor were avoided for ROI placement. In patients with multiple bony or soft tissue tumors, the largest lesion was selected for calculation of the mean ADC value.

Histopathology

Histopathological diagnosis of musculoskeletal tumors was established on surgically resected specimens in 29 patients, core needle biopsy specimens in 41 patients, and fine-needle aspiration (FNAC) specimens in three patients. Ultrasound-guided (USG) or CT-guided core needle biopsy specimens were obtained by using 


\section{Cureus}

a 16-18 gauge core biopsy needle (BARD Biopsy System; Tempe, Arizona, USA). MRI studies were always performed before the biopsy or aspiration procedure. Biopsy or aspiration procedures were done from the enhancing, non-necrotic, and diffusion-restricted portion of a tumor.

Statistical Analysis

All statistical analysis was performed using Statistical Package for Social Sciences (SPSS) version 16 (IBM Corp., Armonk, NY, USA). The clinical data and different parameters of conventional MR imaging in bone and soft tissue tumors were evaluated with a chi-square test. The strength of association between the mean $\mathrm{ADC}$ values with the nature of tumor on histopathology was assessed using an independent t-test and oneway analysis of variance (ANOVA) test. Optimal cut-off mean ADC values of various musculoskeletal tumors were obtained from receiver operating characteristic (ROC) curve analysis.

\section{Results}

Seventy-three histologically proven patients of musculoskeletal tumors were included in this hospital-based retrospective study (Figure 1).

Flow chart of study design

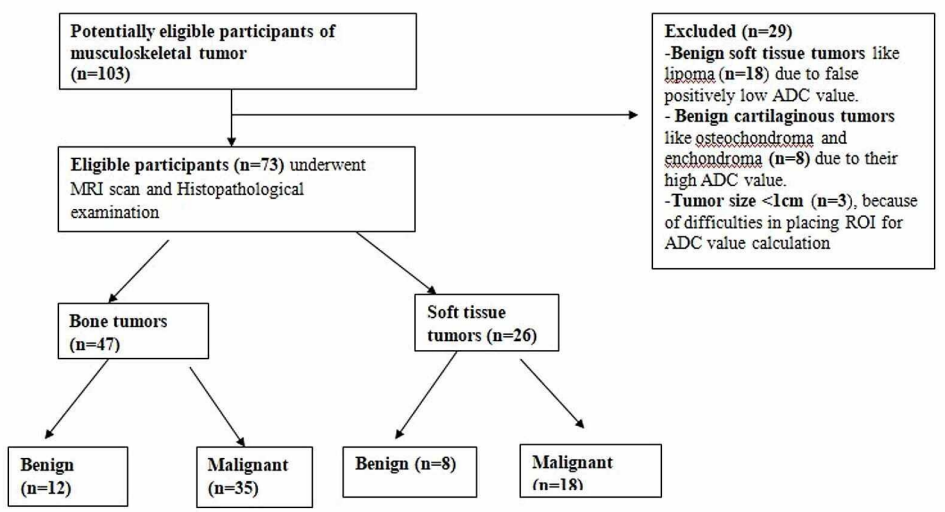

FIGURE 1: Flow chart of study design

The study sample comprised 73 patients of musculoskeletal tumors (bone $=47$, soft tissue $=26$ ). The various histopathological types of bone and soft tissue tumors are shown in Table 2. 


\section{Cureus}

\begin{tabular}{|c|c|c|c|}
\hline Benign tumors $(n=20)$ & Number $(n=20)$ & Malignant tumors $(n=53)$ & Number(n=53) \\
\hline Giant cell tumor of bone & 11 & Bony metastasis & 14 \\
\hline Schwannoma & 5 & Soft tissue sarcoma & 10 \\
\hline Fibromatosis & 2 & Osteosarcoma & 5 \\
\hline Chondromyxoid fibroma & 1 & Fibrosarcoma & 5 \\
\hline \multirow[t]{8}{*}{ Fibroma } & 1 & Malignant fibrous histiocytoma & 5 \\
\hline & & Ewing sarcoma & 4 \\
\hline & & Liposarcoma & 3 \\
\hline & & Lymphoma1 & 2 \\
\hline & & Chondrosarcoma & 2 \\
\hline & & Ameloblastoma & 1 \\
\hline & & Plasmocytoma & 1 \\
\hline & & Malignant Haemangiopericytoma & 1 \\
\hline
\end{tabular}

TABLE 2: Types of bone and soft tissue tumors in 73 patients

The anatomical locations of the tumors are shown in Table 3.

\begin{tabular}{|c|c|c|c|}
\hline Nature of tumor & Anatomical location & Benign & Malignant \\
\hline \multirow{4}{*}{ Bone tumor } & Lower extremity & 9 & 20 \\
\hline & Upper extremity & 1 & 7 \\
\hline & Buttock and pelvic area & 1 & 7 \\
\hline & Back and chest wall & - & 1 \\
\hline \multirow[t]{4}{*}{ Soft tissue tumor } & Lower extremity & 2 & 8 \\
\hline & Upper extremity & 1 & 9 \\
\hline & Buttock and pelvic area & 2 & 0 \\
\hline & Back and chest wall & 3 & 1 \\
\hline
\end{tabular}

TABLE 3: The anatomical location of various bone and soft tissue tumors in 73 patients.

\section{Results of clinical data and conventional MR images}

Patient age, tumor margin, tumor necrosis, and adjacent joint involvement were found to be significantly related to the ability to differentiate benign and malignant bone tumors, but gender, neurovascular bundle involvement, peri-tumoral edema, and tumor heterogeneity were not (Table 4). 


\section{Cureus}

\begin{tabular}{|c|c|c|c|c|}
\hline \multicolumn{2}{|l|}{ Parameters } & Benign tumors $(n=12)$ & Malignant tumors $(n=35)$ & p-value \\
\hline \multicolumn{2}{|l|}{ Age (years) } & $29.4 \pm 8.8[\mathrm{SD}]$ & $43.8 \pm 2.1[\mathrm{SD}]$ & 0.001 \\
\hline \multicolumn{2}{|l|}{ Sex } & $M: F=7: 5$ & $M: F=25: 10$ & 0.473 \\
\hline \multicolumn{2}{|l|}{ Tumor size $(\mathrm{cm})$} & $8.3 \pm 5.6[\mathrm{SD}]$ & $11 \pm 5.8[S D]$ & 0.001 \\
\hline \multirow{4}{*}{ Tumor margin } & & & & 0.001 \\
\hline & well- derined & $6(50 \%)$ & $3(8.6 \%)$ & \\
\hline & Partially ill-defined & $5(41.7 \%)$ & $20(57.1 \%)$ & \\
\hline & III -defined & $1(8.3 \%)$ & $12(34.3 \%)$ & \\
\hline \multirow{4}{*}{ Neurovascular bundle (NVB) } & & & & 0.146 \\
\hline & No involvement & $4(33.3 \%)$ & $10(28.6 \%)$ & \\
\hline & Displacement & $7(58.3 \%)$ & $16(45.7 \%)$ & \\
\hline & Encasement & $1(8.3 \%)$ & $9(25.7 \%)$ & \\
\hline \multirow{3}{*}{ Peri-tumoral edema } & & & & 0.096 \\
\hline & Yes & $7(58.3 \%)$ & $33(94.3 \%)$ & \\
\hline & No & $5(41.7 \%)$ & $2(5.7 \%)$ & \\
\hline \multirow{3}{*}{ Tumor Heterogeneity } & & & & 0.569 \\
\hline & Yes & $7(58.3 \%)$ & $30(85.7 \%)$ & \\
\hline & No & $5(41.7 \%)$ & $5(14.3 \%)$ & \\
\hline \multirow{3}{*}{ Tumor Necrosis } & & & & 0.044 \\
\hline & Yes & $4(33.3 \%)$ & $23(65.7 \%)$ & \\
\hline & No & $8(66.7 \%)$ & $12(34.3 \%)$ & \\
\hline \multirow{3}{*}{ Adjacent joint involvement } & & & & 0.007 \\
\hline & No involvement & $5(41.7 \%)$ & $21(60 \%)$ & \\
\hline & Involvement & $7(58.3 \%)$ & $13(37.1 \%)$ & \\
\hline
\end{tabular}

TABLE 4: showed the results of clinical data and Conventional MR imaging findings in bone tumors

Patient age, tumor size, margin, peri-tumoral edema, neurovascular bundle involvement, tumor heterogeneity, and tumor necrosis showed statistical significance to differentiate benign from malignant soft tissue tumors (Table 5). 


\section{Cureus}

\begin{tabular}{|c|c|c|c|c|}
\hline \multicolumn{2}{|l|}{ Parameters } & Benign tumors $(n=8)$ & Malignant tumors $(n=18)$ & p-value \\
\hline \multicolumn{2}{|l|}{ Age (years) } & $25.7 \pm 14.8[S D]$ & $37.8 \pm 16.8[\mathrm{SD}]$ & 0.001 \\
\hline \multicolumn{2}{|l|}{ Sex } & $\mathrm{M}: \mathrm{F}=3: 5$ & $M: F=9: 9$ & 0.083 \\
\hline \multicolumn{2}{|l|}{ Tumor size(cm) } & $9.4 \pm 4[S D]$ & $10 \pm 5.9[S D]$ & 0.001 \\
\hline \multirow{3}{*}{ Tumor margin } & & & & 0.001 \\
\hline & Partially ill-defined & $3(37.5 \%)$ & $8(44.4 \%)$ & \\
\hline & III -defined & & $5(27.8 \%)$ & \\
\hline \multirow{3}{*}{ Location } & & & & 0.001 \\
\hline & Deep & $5(62.5 \%)$ & $8(44.4 \%)$ & \\
\hline & Both & $2(25 \%)$ & $7(38.9 \%)$ & \\
\hline \multirow{4}{*}{ Neurovascular bundle (NVB) } & & & & 0.029 \\
\hline & No involvement & $3(37.5 \%)$ & $3(16.7 \%)$ & \\
\hline & Displacement & $5(62.5 \%)$ & $9(50 \%)$ & \\
\hline & Encasement & & $6(33.3 \%)$ & \\
\hline \multirow{3}{*}{ Peri-tumoral edema } & & & & 0.032 \\
\hline & Yes & $2(25 \%)$ & $9(50 \%)$ & \\
\hline & No & $6(75 \%)$ & $9(50 \%)$ & \\
\hline \multirow{3}{*}{ Tumor Heterogeneity } & & & & 0.032 \\
\hline & Yes & $2(25 \%)$ & $9(50 \%)$ & \\
\hline & No & $6(75 \%)$ & $9(50 \%)$ & \\
\hline \multirow{3}{*}{ Tumor Necrosis } & & & & 0.001 \\
\hline & Yes & & 6(33.3\%) & \\
\hline & No & $8(100 \%)$ & $12(66.7 \%)$ & \\
\hline \multirow{3}{*}{ Bone involvement } & & & & 0.003 \\
\hline & No involvement & $6(75 \%)$ & $13(72.2 \%)$ & \\
\hline & Involvement & $2(25 \%)$ & $5(27.8 \%)$ & \\
\hline
\end{tabular}

TABLE 5: showed the results of clinical data and Conventional MR imaging findings in soft tissue tumors

Table 4 and Table 5 summarize the clinical data and conventional MRI findings in various bone and soft tissue tumors. Bone tumors were observed in 47 patients (benign=12, malignant=35) and soft tissue tumors in 26 patients (benign=eight, malignant=18). Out of 73 patients, 14 patients had metastatic tumors (Figure 2), 11 patients giant cell tumors (GCT) (Figure 3), 10 patients soft tissue sarcoma, five patients each had osteosarcoma (Figure 4), fibrosarcoma (Figure 5), malignant fibrous histiocytoma (Figure 6) and schwannoma, four patients had Ewings sarcoma, three patients had liposarcoma, two patients each had lymphoma (Figure 7), chondrosarcoma and fibromatosis, and one patient each had haemangioendothelioma, chondromyxoid fibroma (Figure 8), plasmocytoma, malignant haemangiopericytoma and fibroma. 


\section{Cureus}
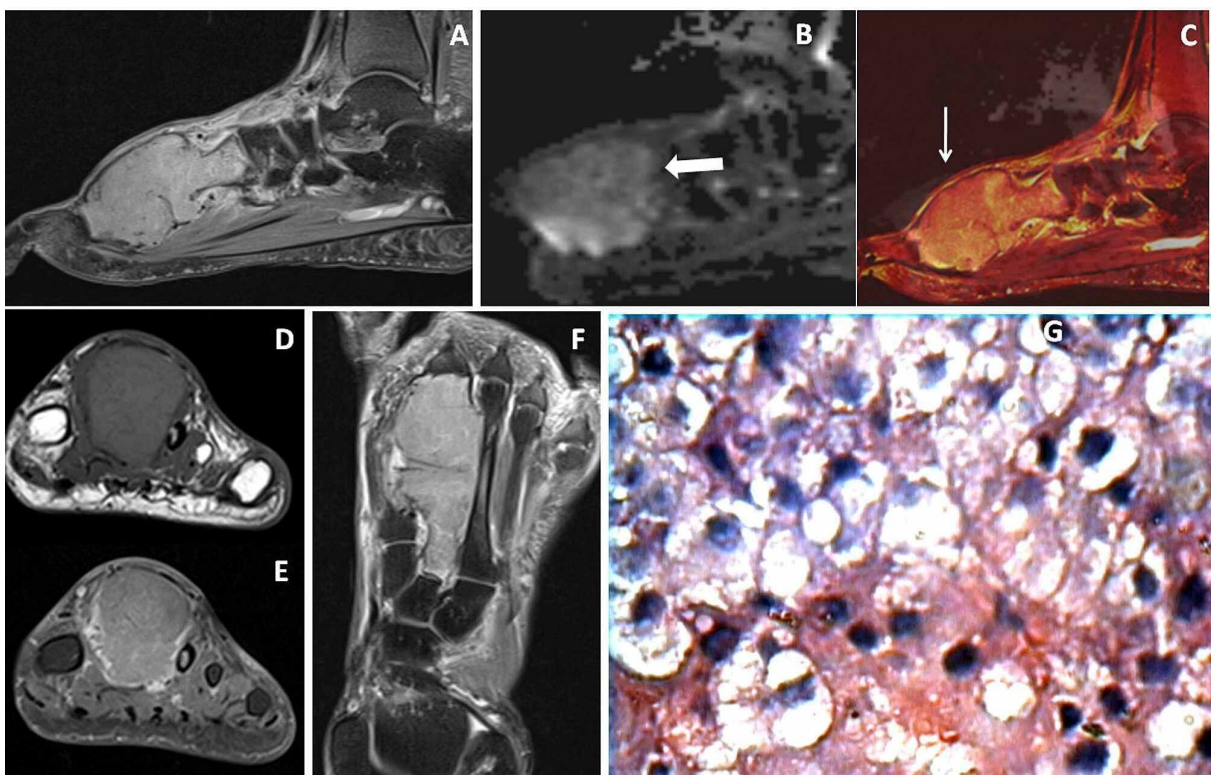

\section{FIGURE 2: Acral metastasis}

Sixty-six-year-old male had swelling in his left foot. Sagittal proton density fat-suppressed (PDFS) image (A) showed a hyperintense expansile mass lesion in the second metatarsal bone extending into articular surfaces. Sagittal diffusion-weighted image (DWI) (B) showed diffusion restriction ( $\leftarrow$ block arrow) with bright signals on fusion image (C) between sagittal PDFS and DWI ( $\downarrow$ arrow). Coronal T1 weighted (T1W) image (D) showed expansion of the affected secondary metatarsal with pressure erosion over the third metatarsal bone. Coronal and axial post gadolinium fat-suppressed T1W images (E\&F) showed moderate homogenous enhancement of the mass. 40X HPE (image G) showed metastasis.
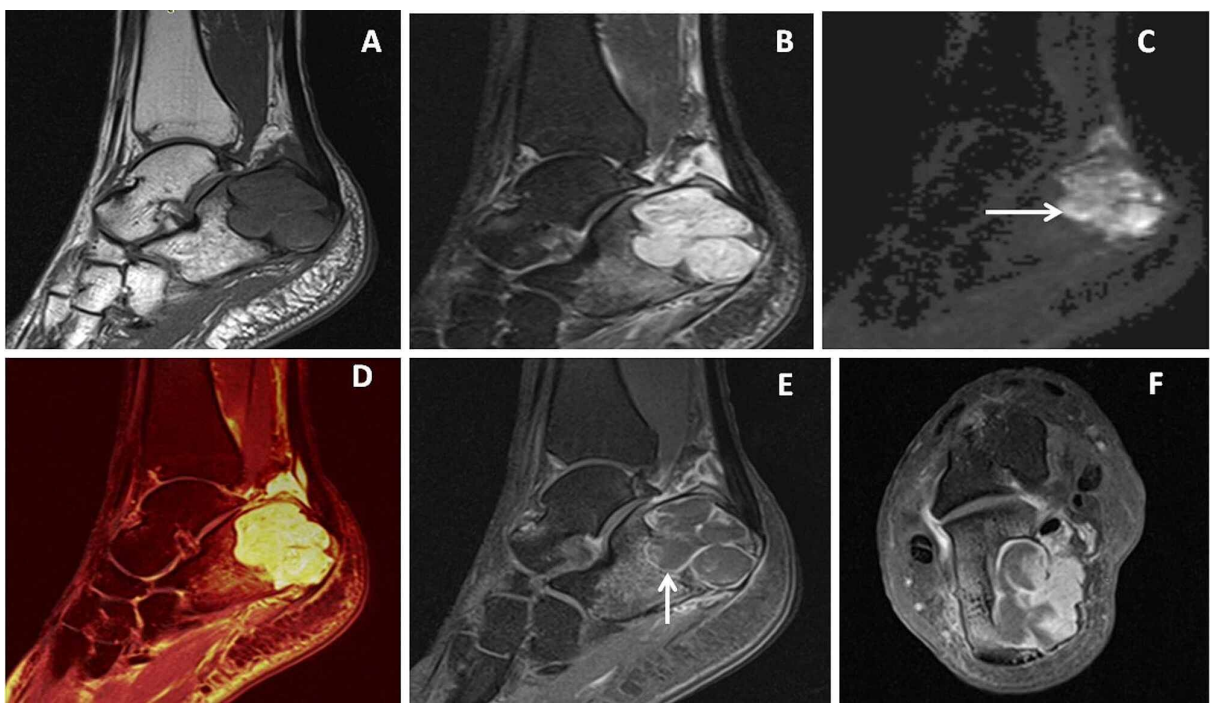

\section{FIGURE 3: Calcaneal giant cell tumor (GCT)}

Thirty-six-year-old female with calcaneal GCT. Sagittal T1 weighted image (T1WI) and proton density fatsuppressed (PDFS) images (A \&B) showed T1WI hypo and PDFS hyperintense expansile lesion in the posterior portion of calcaneum. Sagittal diffusion-weighted imaging (DWI) (C) showed diffusion restriction $\rightarrow$ arrow), where fusion image (D) between sagittal PDFS and DWI showed bright signals within the lesion. Post gadolinium fat-suppressed sagittal and axial T1W images (E \&F) showed moderate heterogeneous enhancement of the mass with more irregular peripherally enhancing solid components with enhancing septae ( $\uparrow$ arrow ). 


\section{Cureus}
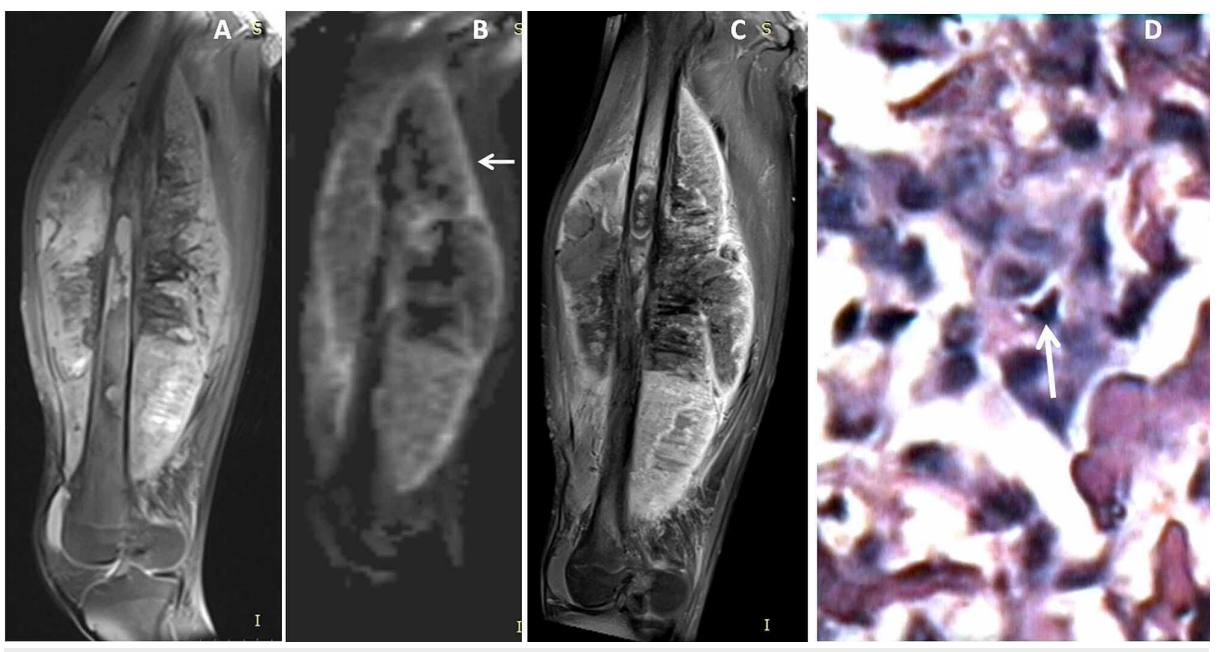

FIGURE 4: Diaphyseal osteosarcoma

Eighteen-year-old male with right thigh swelling. Sagittal proton density fat-suppressed (PDFS) image (A) showed a large lobulated mixed signal intensity mass lesion around the femoral diaphysis with intramedullary altered signal intensities and hypointense sunray appearing new bone formations. Sagittal diffusion-weighted imaging (DWI) (B) showed peripheral dominant diffusion restriction ( $\leftarrow$ arrow). Fat-suppressed post gadolinium sagittal T1 weighted (T1W) image $(C)$ showed heterogeneous enhancement of the mass with areas of necrosis and sunray appearing new bone formation had adjacent soft tissue infiltrations. 40X HPE image (D) showed pleomorphic cells ( $\uparrow$ arrow) with osteoid formations suggesting osteosarcoma.
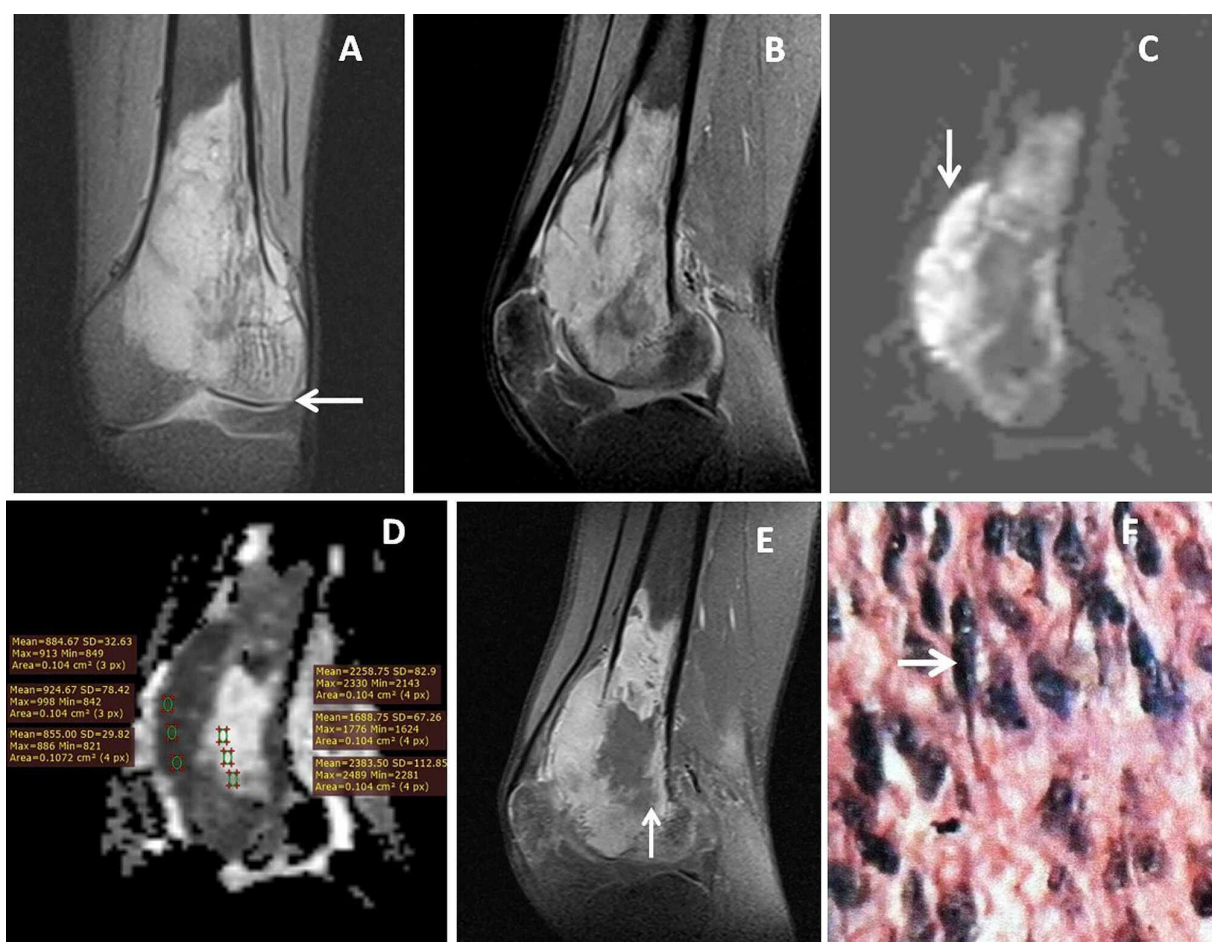

\section{FIGURE 5: Distal femoral fibrosarcoma}

Twenty-year-old male had swelling around the left knee joint. Coronal and sagittal fat-suppressed proton density (PDFS) image (A \& B) showed eccentric hyperintensities in distal meta-diaphyseal region of femur with epiphyseal extension into lateral femoral condyle $(\leftarrow$ arrow) with destruction of cortices. Sagittal diffusion-weighted imaging (DWI) image (C) with b value $1000 \mathrm{~s} / \mathrm{mm} 2$ showed irregular patchy peripheral diffusion restrictions ( $\downarrow$ arrow) where apparent diffusion coefficient (ADC) image (D) showed low ADC value in peripheral and high in central portion of the tumor. Post-gadolinium image (E) showed heterogeneous enhancement of the mass with central necrosis ( $\uparrow$ arrow). 40X HPE image (F) showed highly pleomorphic cells ( $\rightarrow$ arrow) with features of fibrosarcoma. 


\section{Cureus}
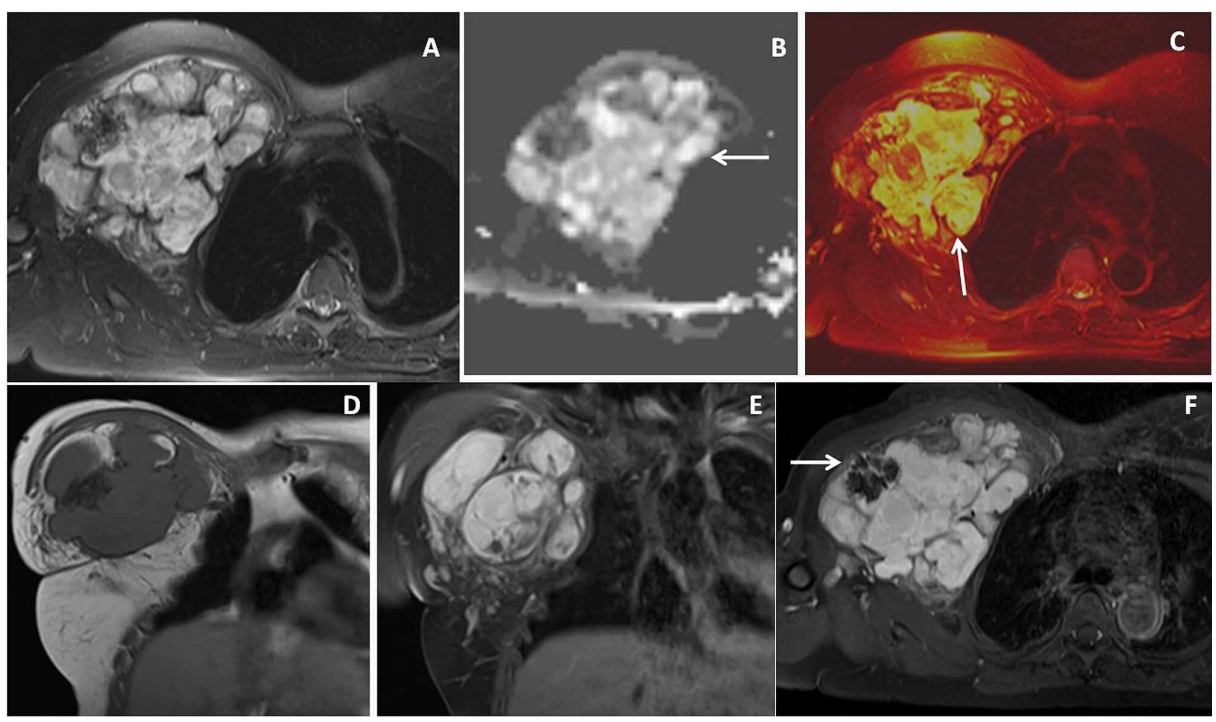

\section{FIGURE 6: Chest wall malignant fibrous histiocytoma}

Thirty-six-year-old female with malignant fibrous histiocytoma (MFH) of right chest wall. Axial proton density fat-suppressed (PDFS) image (A) showed a larger lobulated heterogeneously hyperintense mass lesion in the right upper anterior chest wall causes anterior displacement of the pectoralis muscle. Axial diffusionweighted image (DWI) (B) image shows bright signals within the mass $(\leftarrow$ arrow) while fusion image (C) between axial PDFS and DWI showed the bright signals in major portions of lesion ( $\uparrow$ arrow). Coronal T1 weighted image (T1WI) (D) shows T1WI isointense signal intensities of lesion with ill-defined T1W hypointensities within. Fat-suppressed post gadolinium coronal and axial T1W images (E \& F) showed heterogeneous enhancement of the lesion with non-enhancing T1 low signal intensity lesion( $\rightarrow$ arrow).

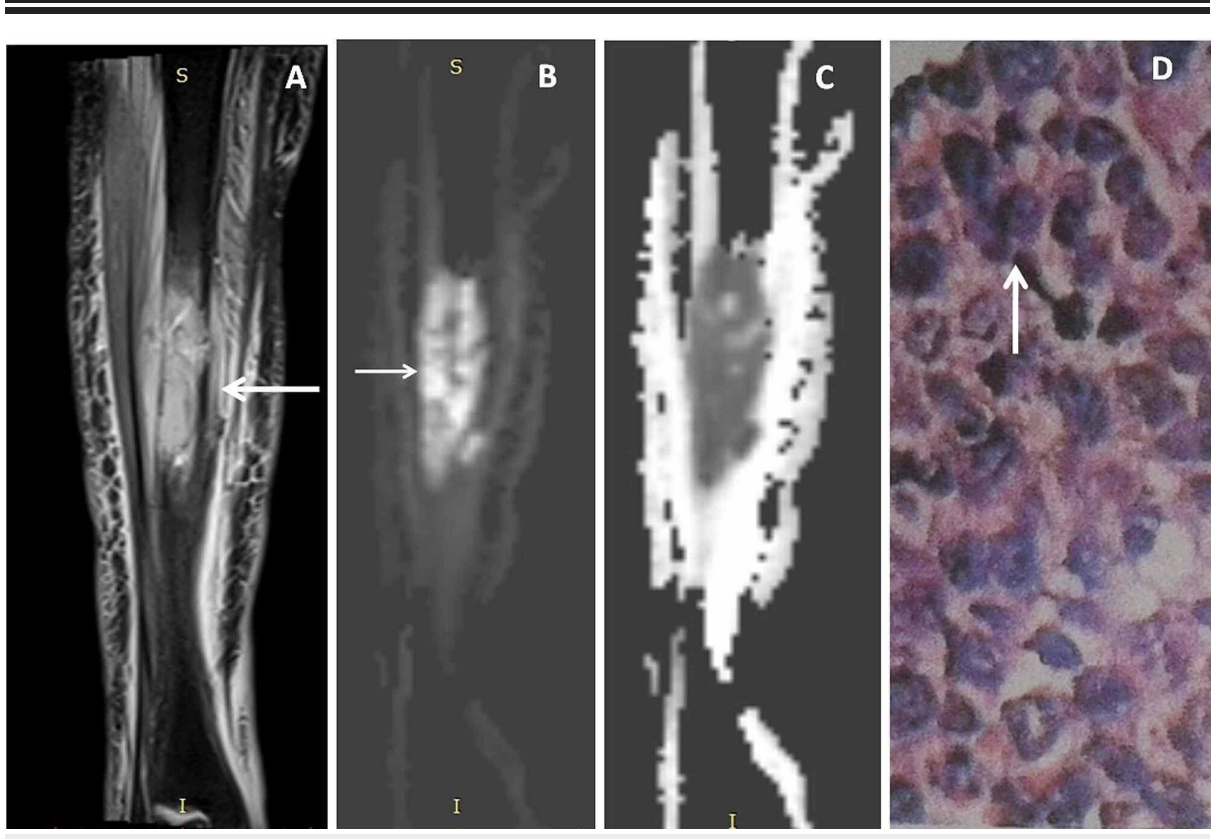

\section{FIGURE 7: Tibial shaft lymphoma}

Forty-seven-year-old female with right leg swelling. Coronal proton density fat-suppressed (PDFS) image (A) showed minimally expansile intramedullary lesion in middle $3 \mathrm{rd}$ of tibial shaft with destructions of bony cortices and surrounding soft tissue infiltrations ( $\leftarrow$ arrow). Coronal diffusion-weighted image (DWI) (B) and apparent diffusion coefficient (ADC) map image $(C)$ showed diffusion restriction $(\rightarrow$ arrow) with low ADC value. 40X HPE image (D) showed bony tissue infiltrated by tumor composed of small round to oval cells, ( $\uparrow$ arrow) where the cells showed vesicular nuclei with prominent nucleoli. Immunehistochemistry confirmed diagnosis of non-Hodgkin's lymphoma with positive leucocyte common antigen (LCA) and negative cytokeratin and desmine. 


\section{Cureus}
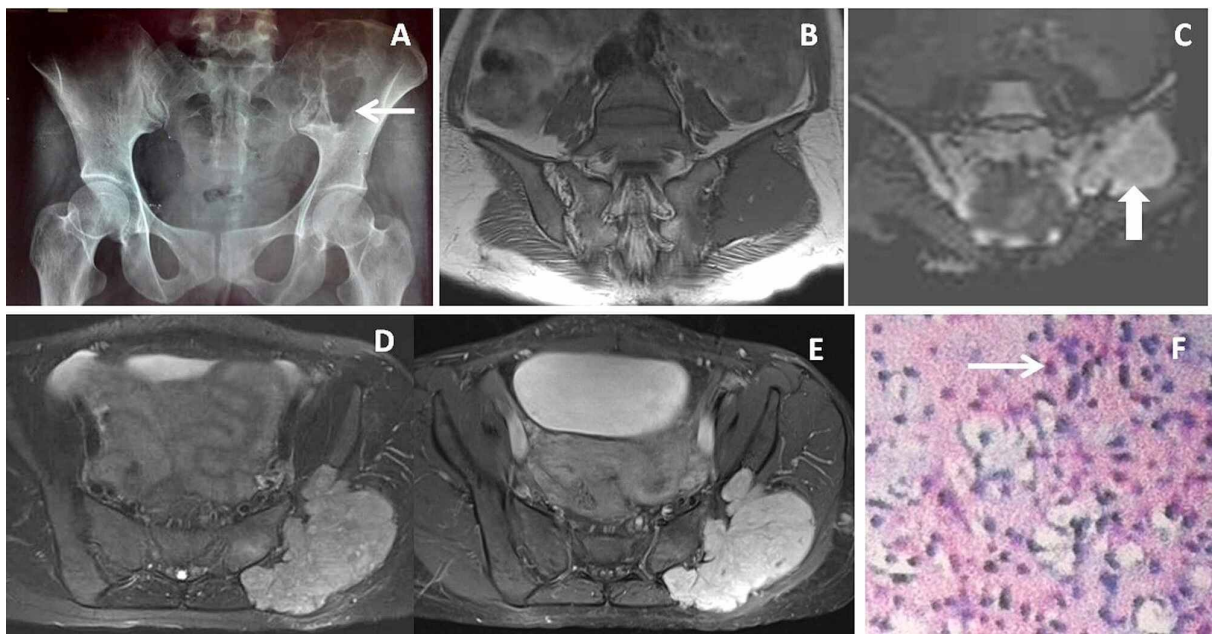

\section{FIGURE 8: lliac bone chondromyxoid fibroma}

Thirty-two-year-old female presented with pain and swelling in left buttock for two years. X-ray AP view of pelvis (A) showed ill-defined osteolytic lesion in left iliac bone ( $\leftarrow$ arrow). Coronal T1 weighted (T1W) image $B$ ) showed a well-defined lobulated hypointense lesion in the left iliac blade. Coronal diffusion-weighted image (DWI) (C) shows diffusion restriction within the mass ( $\uparrow$ arrow). Axial proton density fat-suppressed (PDFS) image (D) showed the lobulated hyperintense lesion in the left iliac blade displacing adjacent gluteal muscles. Post gadolinium axial T1W image $(E)$ showed moderate homogenous enhancement of the lesion. 10X HPE image $(F)$ showed lobules of spindle-shaped cells in abundant myxoid to chondroid stroma ( $\rightarrow$ arrow) suggesting chondromyxoid fibroma.

\section{Results of quantitative diffusion-weighted imaging}

The mean ADC value of bone tumor was $0.996 \pm 0.269[\mathrm{SD}] \mathrm{x} 10-3 \mathrm{~mm} 2 / \mathrm{s}$ and soft tissue tumor was $1.216 \pm$ $0.390[\mathrm{SD}] \mathrm{x} 10-3 \mathrm{~mm} 2 / \mathrm{s}$. There was a statistically significant difference between the mean ADC value of bone and soft tissue tumors ( $\mathrm{p}$-value 0.006 ) using the unpaired student $\mathrm{t}$-test. The mean $\mathrm{ADC}$ value of benign bone tumor was $1.257 \pm 0.327[\mathrm{SD}] \mathrm{x} 10-3 \mathrm{~mm} 2 / \mathrm{s}$ and malignant bone tumor was $0.951 \pm 0.177[\mathrm{SD}] \mathrm{x} 10$ $3 \mathrm{~mm} 2 / \mathrm{s}$ with a statistically significant difference between the mean ADC value of benign and malignant bone tumors (p-value 0.001). The mean ADC value of benign soft tissue tumor was $1.603 \pm 0.444$ [SD] x 10$3 \mathrm{~mm} 2 / \mathrm{s}$ and malignant soft tissue tumor was $1.036 \pm 0.186[\mathrm{SD}] \times 10-3 \mathrm{~mm} 2 / \mathrm{s}$ with a statistically significant difference between the mean ADC value of benign and malignant soft tissue tumors (p-value 0.01) using the unpaired student $t$-test. The distribution of the mean ADC value of bone and soft tissue tumors is shown in the boxplot (Figure 9).

(a)

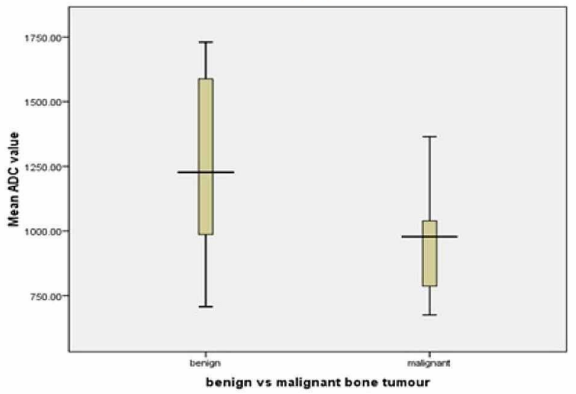

(b)

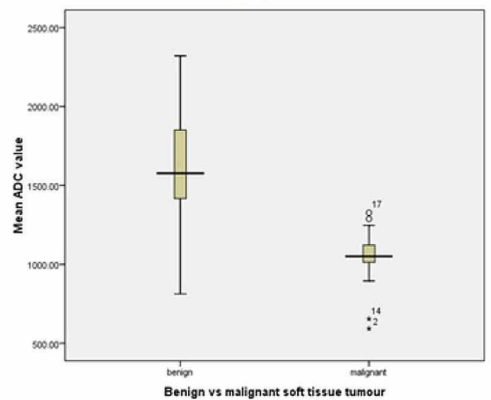

FIGURE 9: Boxplot summarizing the range of distribution of the mean apparent diffusion coefficient (ADC) values of bone and soft tissue tumors in 73 patients.

(a) shows a boxplot of mean ADC value of benign and malignant bone tumors show a considerable overlap between these two groups. Despite this overlap, there was a statistically significant difference between the mean ADC value of benign and malignant bone tumors with a p-value of 0.001 . (b) shows a boxplot of mean $A D C$ value of benign and malignant soft tissue tumors with a statistically significant difference between the mean $A D C$ value with a $p$-value of 0.001 . 


\section{Cureus}

Table 6 shows the mean ADC value of musculoskeletal tumors in our study sample.

\begin{tabular}{|c|c|c|}
\hline Tumor & Number (\%) & Mean ADC (x 10-3 mm2/s) \\
\hline Bone Tumors & 47/73(64.4\%) & $0.996 \pm 0.269[\mathrm{SD}]$ \\
\hline Soft tissue tumor & 26/73(35.6\%) & $1.216 \pm 0.390[\mathrm{SD}]$ \\
\hline Benign bone tumor & $12 / 47(25.5 \%)$ & $1.257 \pm 0.327[\mathrm{SD}]$ \\
\hline Malignant bone tumor & $35 / 47(74.5 \%)$ & $0.951 \pm 0.177[\mathrm{SD}]$ \\
\hline Benign soft tissue tumor & $8 / 26(30.8 \%)$ & $1.603 \pm 0.444[S D]$ \\
\hline Malignant soft tissue tumor & 18/26(69.2\%) & $1.036 \pm 0.186[S D]$ \\
\hline Bony Metastasis & $14 / 73(19.2 \%)$ & $0.939 \pm 0.109[\mathrm{SD}]$ \\
\hline Soft tissue sarcoma & 10/73(13.7\%) & $1.081 \pm 0.234[\mathrm{SD}]$ \\
\hline Giant cell tumor (GCT) & 11/73(15.1\%) & $1.251 \pm 0.342[\mathrm{SD}]$ \\
\hline Osteosarcoma & $5 / 73(6.8 \%)$ & $0.782 \pm 0.125[\mathrm{SD}]$ \\
\hline Malignant Fibrous Histiocytoma & $5 / 73(6.8 \%)$ & $0.916 \pm 0.159[S D]$ \\
\hline Fibrosarcoma & $5 / 73(6.8 \%)$ & $0.938 \pm 0.162[S D]$ \\
\hline Ewing sarcoma & $4 / 73(5.5 \%)$ & $0.689 \pm 0.087[\mathrm{SD}]$ \\
\hline Liposarcoma & $3 / 73(4.1 \%)$ & $1.125 \pm 0.029[\mathrm{SD}]$ \\
\hline Schwannoma & $5 / 73(6.8 \%)$ & $1.440 \pm 0.363[\mathrm{SD}]$ \\
\hline Chondrosarcoma & $2 / 73(2.7 \%)$ & $1.324 \pm 0.048[\mathrm{SD}]$ \\
\hline Lymphoma & $2 / 73(2.7 \%)$ & $0.747 \pm 0.315[\mathrm{SD}]$ \\
\hline
\end{tabular}

TABLE 6: summarized the mean apparent diffusion coefficient (ADC) value of commonly encountered musculoskeletal tumors in 73 patients.

\section{ROC curve analysis of ADC mapping}

Receiver operating characteristic (ROC) curve analysis showed cut-off mean ADC value of 1.058 x 10$3 \mathrm{~mm} 2 / \mathrm{s}$ for differentiating benign from malignant bone tumor with a sensitivity of $83.3 \%$, specificity of $66.7 \%$, positive predictive value of $87.8 \%$, negative predictive value of $57.1 \%$ and a diagnostic accuracy of 78.7\% (Figure 10). 


\section{Cureus}

ROC Curve

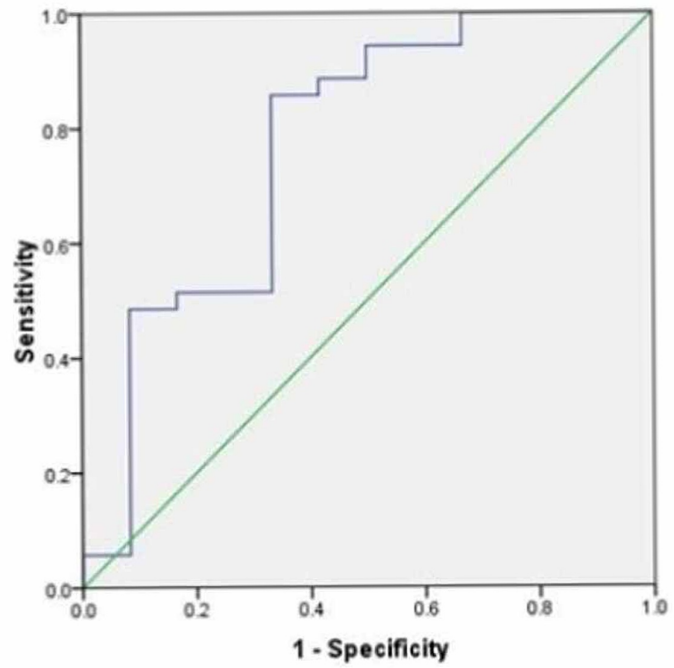

\begin{tabular}{|c|c|c|}
\hline $\begin{array}{l}\text { Criteria (Cut off } A D C \\
\text { value) }\end{array}$ & Sensitivity(\%) & Specificity(\%) \\
\hline 50.982 & 51.1 & 83.3 \\
\hline$\leq 1.025$ & 68.6 & 66.7 \\
\hline$\leq 1.058$ & 82.9 & 66.7 \\
\hline
\end{tabular}

FIGURE 10: Receiver operating characteristic (ROC) curve of mean apparent diffusion coefficient (ADC) value of bone tumors in 47 patients.

However four patients (8.5\%) of giant cell tumor (GCT) showed ADC value lower than the cut-off value of $1.058 \times 10-3 \mathrm{~mm} 2 / \mathrm{s}$ while another one patient (2.1\%) of chondrosarcoma showed ADC value higher than the cut-off ADC value. Receiver operating characteristic (ROC) curve analysis showed cut off mean ADC value of $1.198 \times 10-3 \mathrm{~mm} 2 / \mathrm{s}$ for differentiating benign from malignant soft tissue tumor with a sensitivity of $83.3 \%$, specificity of $87.5 \%$, positive predictive value of $93.7 \%$, negative predictive value of $70 \%$ and a diagnostic accuracy of $84.6 \%$ (Figure 11 ).

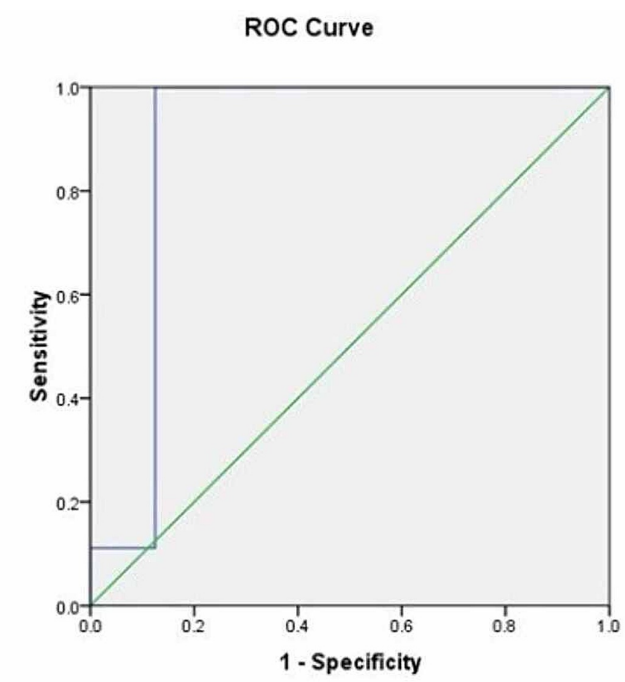

\begin{tabular}{|l|l|l|}
\hline Criteria (Cut off ADC value) & Sensitivity (\%) & Specificity (\%) \\
\hline$\leq 1.073$ & 61.1 & 87.5 \\
\hline$\leq 1.135$ & 77.8 & 87.5 \\
\hline$\leq 1.198$ & 83.3 & 87.5 \\
\hline
\end{tabular}

FIGURE 11: Receiver operating characteristic (ROC) curve of mean 
However two patients (7.7\%) of soft tissue sarcoma showed ADC value higher than the cut-off ADC value of $1.198 \times 10^{-3} \mathrm{~mm} 2 / \mathrm{s}$ while another one patient (3.8\%) of schwannoma showed ADC value lower than cut-off ADC value.

\section{Discussion}

Quantitative diffusion-weighted imaging (DWI) and ADC mapping had a valuable role in characterizing and differentiating various bone and soft tissue tumors, which may improve the diagnostic accuracy in addition to the conventional MR imaging [14]. The non-contrast MRI techniques like DWI and MR Spectroscopy can be used in situations where intravenous contrast media is contraindicated. Usually, malignant tumors have low $\mathrm{ADC}$ values and benign tumors have high $\mathrm{ADC}$ values with some exceptions like giant cell tumor (GCT) and osteoblastoma, which show low ADC values [14]. The low ADC values in GCTs are due to the reduction of extracellular space from histiocytes, multi-nucleated giant cells, hemosiderin granules, and collagenous strands [3,16-17]. High ADC values were observed in chondroid lesions, probably due to high free extracellular water content, regardless of their cellularity and histological grading [18-20]. The malignant chondroid tumors usually show higher ADC values than benign chondroid tumors [17] because of the high content of the chondroid matrix [16-17]. Ewing sarcoma shows the lowest ADC value in the group of sarcomas [21].

Quantitative ADC mapping helps in differentiating residual or recurrent tumor in post-treated (postirradiated) or post-operated musculoskeletal tumors [22]. Post-treated tumors with areas of higher ADC value than the pretreatment value indicate tumor cell necrosis which suggests a positive response to the therapy $[6,22]$. Some previous studies showed that post-treatment increasing ADC value in primary bone sarcoma correlated well with successful treatment [7,23].

In our study, conventional MR imaging parameters like tumor margin, tumor necrosis, and adjacent joint involvement were found to be significantly related to the ability to differentiate benign and malignant bone tumors, but neurovascular bundle involvement, peritumoral edema, and tumor heterogeneity were not. But most of the conventional MRI parameters like tumor size, margin, peri-tumoral edema, neurovascular bundle involvement, tumor heterogeneity, and tumor necrosis showed statistical significance to differentiate benign from malignant soft tissue tumor. So quantitative DWI imaging helps as an additional reliable tool in the evaluation of musculoskeletal tumors, especially bone tumors. When low ADC values and malignant MRI characteristics are combined, more accurate results are obtained in our study sample as compared to evaluating only ADC values.

In our study sample, considerable ADC value overlap was noted while differentiating various benign and malignant bone as well as soft tissue tumors. Even though in our study sample, 29 (82.8\%) out of 35 patients of malignant bone tumor showed lower ADC value below the cut-off value of $1.058 \times 10-3 \mathrm{~mm} 2 / \mathrm{s}$ while 15 (83.3) out of 18 patients of malignant soft tissue tumor showed lower ADC value below the cut-off value of $1.198 \times 10-3 \mathrm{~mm} 2 / \mathrm{s}$. But we do not know whether this ADC value overlapping is mainly due to heterogeneity of various bone and soft tissue tumor subtypes or other factors. However, our study findings of cut-off ADC values add further support to previously published studies [6,24]. Our study sample showed a cut-off mean $\mathrm{ADC}$ value of $1.198 \times 10-3 \mathrm{~mm} 2 / \mathrm{s}$ for differentiating benign and malignant soft tissue tumor with a sensitivity of $83.3 \%$, specificity of $87.5 \%$ and these findings are well correlated with the previous study of Choi et al. [25] who found cut-off mean ADC value of $1.18 \times 10-3 \mathrm{~mm} 2 / \mathrm{sec}$ with a sensitivity of $86.11 \%$ and specificity of $77.05 \%$, Hassanien et al. who found cut-off ADC value of $1.235 \times 10-3 \mathrm{~mm} 2 / \mathrm{s}$ with a sensitivity of $73 \%$ and specificity of $91.7 \%$ [26], and Robba et al. [27] who found cut-off ADC value of $1.45 \times 10-3 \mathrm{~mm} 2 / \mathrm{s}$ with a sensitivity of $90.9 \%$ and specificity of $60 \%$.

A study conducted by Geneidi et al. with the inclusion of vertebral lesions showed cut-off ADC value of 0.67 x $10-3 \mathrm{~mm} 2 / \mathrm{sec}$ for differentiating benign and malignant bone tumor with sensitivity of $94 \%$ and specificity of $79 \%$ [28] while in our study sample without inclusion of vertebral lesions showed cut off mean ADC value of $1.074 \times 10-3 \mathrm{~mm} 2 / \mathrm{s}$ with a sensitivity of $83.3 \%$, specificity of $72.7 \%$. In our study sample, four patients (8.5\%) of GCT showed false positivity for malignant bone tumors with ADC value below the cut-off ADC value of $1.058 \times 10-3 \mathrm{~mm} 2 / \mathrm{s}$. Ashikyan et al. [29] showed GCT had a mean ADC value of 1.0 $\pm 0.2[\mathrm{SD}] \mathrm{x} 10-$ $3 \mathrm{~mm} 2 / \mathrm{s}$ while in our study sample it was $1.251 \pm 0.342[\mathrm{SD}]$.

Neubauer et al. [30] observed the mean ADC value below $1.03 \times 10-3 \mathrm{~mm} 2 / \mathrm{s}$ was a strong indicator for pediatric musculoskeletal malignancy after using a b-value of 50 and $800 \mathrm{~s} / \mathrm{mm} 2$. In our study sample, we found mean ADC value below $1.058 \times 10-3 \mathrm{~mm} 2 / \mathrm{s}$ is a good indicator of malignant bone tumor with a sensitivity of $83.3 \%$, specificity of $66.7 \%$, and diagnostic accuracy of $78.7 \%$. So our findings are well correlated with this previous study. 


\section{Limitation}

Exclusion of various benign bone as well as soft tumors in our study sample and inclusion of most of giant cell tumor of bone limited the mean ADC value results. Vast heterogeneity of the musculoskeletal tumors is a major weakness for DWI imaging and ADC mapping for diagnosis and differentiation of various musculoskeletal tumors. Therefore, a larger study sample size to confirm these findings is warranted in the future.

\section{Conclusions}

Quantitative diffusion-weighted imaging and ADC mapping helps in the evaluation of musculoskeletal tumors in conjunction with conventional MRI sequences. In our study, we found a statistically significant difference of mean $\mathrm{ADC}$ value between the benign and malignant bone as well as soft tissue tumors. Even though DWI and ADC mapping alone may not be useful for differentiating various benign and malignant musculoskeletal tumors because of overlapping ADC values. However, mean ADC values may serve as an additional tool while combining with the conventional MRI characteristics to diagnose and differentiate various musculoskeletal tumors.

\section{Additional Information \\ Disclosures}

Human subjects: Consent was obtained by all participants in this study. Animal subjects: All authors have confirmed that this study did not involve animal subjects or tissue. Conflicts of interest: In compliance with the ICMJE uniform disclosure form, all authors declare the following: Payment/services info: All authors have declared that no financial support was received from any organization for the submitted work. Financial relationships: All authors have declared that they have no financial relationships at present or within the previous three years with any organizations that might have an interest in the submitted work. Other relationships: All authors have declared that there are no other relationships or activities that could appear to have influenced the submitted work.

\section{References}

1. Kaplan PA, Helms CA, Dussalt R: Tumors. Musculoskeletal MRI. Kaplan PA, Helms CA, Dussalt R, et al. (ed): WB Saunders, Philadelphia; 2001. 23-150.

2. Choi YJ, Lee IS, Song YS, Kim JI, Choi K-U, Song JW: Diagnostic performance of diffusion-weighted (DWI) and dynamic contrast-enhanced (DCE) MRI for the differentiation of benign from malignant soft-tissue tumors. J Magn Reson Imaging. 2019, 50:798-809. 10.1002/jmri.26607

3. Lee SY, Jee WH, Jung JY, et al.: Differentiation of malignant from benign soft tissue tumors: use of additive qualitative and quantitative diffusion-weighted MR imaging to standard MR imaging at 3.0 T. Eur Radiol. 2016, 26:743-754. 10.1007/s00330-015-3878-x

4. Lim HK, Jee WH, Jung JY, et al.: Intravoxel incoherent motion diffusion-weighted MR imaging for differentiation of benign and malignant musculoskeletal tumors at 3 T. Br J Radiol. 2018, 91:20170636-10. 10.1259/bjr.20170636

5. Le Bihan D, Breton E, Lallemand D, Grenier P, Cabanis E, Laval-Jeantet M: MR imaging of intravoxel incoherent motions: application to diffusion and perfusion in neurologic disorders. Radiology. 1986, 161:401-407. 10.1148/radiology.161.2.3763909

6. Khoo MY, Tyler PA, Saifuddin A, Padhani AR: Diffusion-weighted imaging (DWI) in musculoskeletal MRI: a critical review. Skeletal Radiol. 2011, 40:665-681. 10.1007/s00256-011-1106-6

7. Hayashida Y, Yakushiji T, Awai K, et al.: Monitoring therapeutic responses of primary bone tumors by diffusion-weighted image: initial results. Eur Radiol. 2006, 16:2637-2643. 10.1007/s00330-006-0342-y

8. Ogawa M, Kan H, Arai N, Murai T, Manabe Y, Sawada Y, Shibamoto Y: Differentiation between malignant and benign musculoskeletal tumors using diffusion kurtosis imaging. Skeletal Radiol. 2019, 48:285-292. 10.1007/s00256-018-2946-0

9. Uhl M, Saueressig U, Koehler G, et al.: Evaluation of tumor necrosis during chemotherapy with diffusionweighted MR imaging: preliminary results in osteosarcomas. Pediatr Radiol. 2006, 36:1306-1311. 10.1007/s00247-006-0324-X

10. Hayashida Y, Hirai T, Yakushiji T, et al.: Evaluation of diffusion-weighted imaging for the differential diagnosis of poorly contrast-enhanced and T2-prolonged bone masses: Initial experience. J Magn Reson Imaging. 2006, 23:377-382. 10.1002/jmri.20512

11. Yakushiji T, Oka K, Sato H, et al.: Characterization of chondroblastic osteosarcoma: gadolinium-enhanced versus diffusion-weighted MR imaging. J Magn Reson Imaging. 2009, 29:895-900. 10.1002/jmri.21703

12. Daniel Jr III A, Ullah E, Wahab S, Kumar V: Relevance of MRI in the prediction of malignancy of musculoskeletal system-a prospective evaluation. BMC Musculoskelet Disord. 2009, 10:125. 10.1186/14712474-10-125

13. Oh E, Yoon YC, Kim JH, Kim K: Multiparametric approach with diffusion-weighted imaging and dynamic contrast-enhanced MRI: a comparison study for differentiating between benign and malignant bone lesions in adults. Clin Radiol. 2017, 72:552-559. 10.1016/j.crad.2017.02.017

14. Nagata S, Nishimura H, Uchida M, et al.: Diffusion-weighted imaging of soft tissue tumors: usefulness of the apparent diffusion coefficient for differential diagnosis. Radiat Med. 2008, 26:287-295. 10.1007/s11604-0080229-8

15. Subhawong TK, Jacobs MA, Fayad LM: Insights into quantitative diffusion-weighted MRI for musculoskeletal tumor imaging AJR. Am J Roentgenol. 2014, 203:560-572. 10.2214/AJR.13.12165 
16. Costa FM, Ferreira EC, Vianna EM: Diffusion-weighted magnetic resonance imaging for the evaluation of musculoskeletal tumors. Magn Reson Imaging Clin N Am. 2011, 19:159-180. 10.1016/j.mric.2010.10.007

17. Nagata S, Nishimura H, Uchida M, Hayabuchi N: Usefulness of diffusion-weighted MRI in differentiating benign from malignant musculoskeletal tumors. Nihon Igaku Hoshasen Gakkai Zasshi. 2005, 65:30-36.

18. Douis H, Jeys L, Grimer R, Vaiyapuri S, Davies AM: Is there a role for diffusion-weighted MRI (DWI) in the diagnosis of central cartilage tumors?. Skeletal Radiol. 2015, 44:963-969. 10.1007/s00256-015-2123-7

19. Douis H, Singh L, Saifuddin A: MRI differentiation of low-grade from high-grade appendicular chondrosarcoma. Eur Radiol. 2014, 24:232-240. 10.1007/s00330-013-3003-y

20. Albano D, Messina C, Gitto S, Papakonstantinou O, Sconfienza LM: Differential diagnosis of spine tumors: my favorite mistake. Semin Musculoskelet Radiol. 2019, 23:26-35. 10.1055/s-0038-1675551

21. Garrett KM, Kim HK, Stanek J, et al.: MR findings of primary bone lymphoma in a 15-year-old girl: emphasis on diffusion-weighted imaging. Pediatr Radiol. 2011, 41:658-662. 10.1007/s00247-010-1893-2

22. Costa FM, Ferreira EC, Vianna EM: Diffusion-weighted magnetic resonance imaging for the evaluation of musculoskeletal tumors. Magn Reson Imaging Clin N Am. 2011, 19:159-180. 10.1016/j.mric.2010.10.007

23. Uhl M, Saueressig U, van Buiren M, et al.: Osteosarcoma: preliminary results of in vivo assessment of tumor necrosis after chemotherapy with diffusion- and perfusion-weighted magnetic resonance imaging. Invest Radiol. 2006, 41:618-623. 10.1097/01.rli.0000225398.17315.68

24. Razek A, Nada N, Ghaniem M, Elkhamary S: Assessment of soft tissue tumors of the extremities with diffusion echoplanar MR imaging. Radiol Med. 2012, 117:96-101. 10.1007/s11547-011-0709-2

25. Choi YJ, Lee IS, Song YS, et al.: Diagnostic performance of diffusion-weighted (DWI) and dynamic contrastenhanced (DCE) MRI for the differentiation of benign from malignant soft-tissue tumors. J Magn Reson Imaging. 2019, 50:798-809. 10.1002/jmri.26607

26. Hassanien OA, Younes RL, Dawoud RM: Diffusion-weighted MRI of soft tissue masses: can measurement of ADC value help in the differentiation between benign and malignant lesions?. Egypt J Radiol Nucl Med. 2018, 49:681-688. 10.1016/j.ejrnm.2018.04.008

27. Robba T, Chianca V, Albano D, et al.: Diffusion-weighted imaging for the cellularity assessment and matrix characterization of soft tissue tumour. Radiol Med. 2017, 122:871-879. 10.1007/s11547-017-0787-X

28. Geneidi ES, Ali HI, Dola EF: Role of DWI in characterization of bone tumors . Egypt J Radiol Nucl Med. 2016, 47:919-927. 10.1016/j.ejrnm.2016.06.017

29. Ashikyan O, Chalian M, Moore D, et al.: Evaluation of giant cell tumors by diffusion- weighted imagingfractional ADC analysis. Skeletal Radiol. 2019, 48:1765-1773. 10.1007/s00256-019-03219-8

30. Neubauer H, Evangelista L, Hassold N, et al.: Diffusion-weighted MRI for detection and differentiation of musculoskeletal tumorous and tumor-like lesions in pediatric patients. World J Pediatr. 2012, 8:342-349. 10.1007/s12519-012-0379-8 